\title{
Homoclinic and heteroclinic solutions for a class of second-order non-autonomous ordinary differential equations: multiplicity results for stepwise potentials
}

Elisa Ellero and Fabio Zanolin*

Dedicated to Professor Jean Mawhin

${ }^{\text {*Correspondence: }}$ fabio.zanolin@uniud.it Department of Mathematics and Computer Science, University of Udine, Via delle Scienze 206, Udine, 33100, Italy

\begin{abstract}
We prove some multiplicity results for a class of one-dimensional nonlinear Schrödinger-type equations of the form$$
x^{\prime \prime}+2 k x-2 g(t) x^{3}=0,
$$

where $k>0$ and the weight $g(t)$ is a positive stepwise function. Instead of the cubic term, more general nonlinearities can be considered as well.
\end{abstract}

MSC: $34 \mathrm{C} 37 ; 34 \mathrm{~B} 15$

Keywords: homoclinic solutions; heteroclinic solutions; multiplicity; nonlinear Schrödinger equation; stepwise potential; topological methods

\section{Introduction}

This paper deals with the study of homoclinic and heteroclinic solutions for a class of nonlinear second-order differential equations of the form

$$
x^{\prime \prime}+\sigma x-a(t) h(x)=0
$$

where $\sigma>0$ is a fixed (positive) coefficient and $\left.\left.a(\cdot): \mathbb{R} \rightarrow \mathbb{R}_{0}^{+}:=\right] 0,+\infty\right)$ is a bounded weight function. For the nonlinear term, which we split as

$$
h(x)=x f(x)
$$

we suppose that

(*) $f: \mathbb{R} \rightarrow \mathbb{R}^{+}:=[0,+\infty)$ is a locally Lipschitz function which is even, strictly increasing on $[0,+\infty)$ such that $f(0)=0$ and $f(+\infty)=+\infty$.

Examples of functions $f$ which are suitable for our considerations are, for instance, $f(s)=s^{2}$ as in [1], or $f(s)=s^{2}+b s^{4}(b>0)$ as in [2]. In both these cases, the function $f$ is even. In Section 3 we briefly describe how this restriction could be avoided.

(c) 2013 Ellero and Zanolin; licensee Springer. This is an Open Access article distributed under the terms of the Creative Commons Attribution License (http://creativecommons.org/licenses/by/2.0), which permits unrestricted use, distribution, and reproduction in any medium, provided the original work is properly cited.

\section{Springer}


Equations of the form (1.1) naturally arise in the search of particular solutions for some classes of nonlinear Schrödinger equations (NLSE) with inhomogeneous nonlinearities. Typical examples are related to the NLSE

$$
i \hbar \frac{\partial \Psi}{\partial t}=-\frac{\hbar^{2}}{2 m} \Delta \Psi+V_{1}(x) \Psi+V_{2}(x)|\Psi|^{p-1} \Psi, \quad x \in \mathbb{R}^{N},
$$

where $\hbar$ is the Planck constant, $m$ is the particle's mass, $i$ is the imaginary unit, $\Delta$ is the Laplace operator and $p>1$. In literature, solutions of the form

$$
\Psi(t, x)=u(x) \exp \left(i \lambda \hbar^{-1} t\right)
$$

where $\lambda \in \mathbb{R}$ and $u(x)$ is a real-valued function, are called stationary waves. Their search leads to the equation

$$
-\frac{\hbar^{2}}{2 m} \Delta u+\lambda u+V_{1}(x) u-V_{2}(x)|u|^{p-1} u=0, \quad x \in \mathbb{R}^{N}
$$

(see [3]). Usually, the Planck constant and the mass are omitted in (1.3) after rescaling.

In many significant models of NLSE, one-dimensional waves are considered. They are studied, for example, in nonlinear optics, in the theory of ocean rogue waves and for BoseEinstein condensates (just to mention a few cases). For instance, in [1], Belmonte-Beitia and Torres analyzed the one-dimensional equation

$$
-\frac{1}{2} u^{\prime \prime}+\lambda u+g(x) u^{3}=0, \quad x \in \mathbb{R}
$$

which is a particular case of (1.3) with $N=1, p=3$ and $V_{1}=0$. In equation (1.4) the nonlinearity is called inhomogeneous due to the presence of a non-constant weight $V_{2}(x)=g(x)$, which in [1] is assumed to be positive. The sign condition on $g(x)$ implies that nontrivial bounded solutions of (1.4) can exist only for $\lambda<0$ (see [1, Theorem 1]). For this reason, we prefer to set $\lambda=-k$ with $k>0$.

In order to study equation (1.4) or its variants, we are going to follow a dynamical system approach, hence we choose to treat the independent variable (which in the applications has a spatial connotation) as a time variable, via the substitution $x \longleftrightarrow t$. Similarly, for the dependent variable, we make the substitution $u \longleftrightarrow x$. In this way, equation (1.4) reads as

$$
x^{\prime \prime}+2 k x-2 g(t) x^{3}=0
$$

which belongs to the same class of (1.1).

In spite of the apparent simplicity of equation (1.5), a throughout study of its solutions may be a rather difficult task for a general nonconstant weight function $g(t)$. Looking for homoclinic and heteroclinic solutions of (1.5), it will be natural to focus on the behavior of $g(t)$ at $\pm \infty$. In similar situations, various authors have confined their study to the case in which $g(t)$ is asymptotically constant [4-6], or eventually constant $[7,8]$. These assumptions are also justified by the analysis of some physical underlying models, in which a layered structure is present. With this respect, see the introduction in [8], where different 
eventually autonomous cases are listed for related NLSEs arising in nonlinear optics. Examples in which the nonlinear term presents a piecewise constant weight function have been studied also in biological and chemical models. In particular, these situations occur in the theory of wave propagation for reaction-diffusion systems; see, for instance, [9-11]. In the context of equation (1.5), examples in which $g(t)$ is a piecewise constant function have been considered as well (see [12]).

The aim of the present paper is to provide multiplicity results regarding homoclinic and heteroclinic solutions for equation (1.1) under particular assumptions on the weight function $a(t)$. Actually, we suppose that $a(t)$ is an eventually constant piecewise function with only two steps.

Our approach combines phase-plane analysis with time-mapping estimates. As in [5, 7, $8,10,11,13]$, the solutions are obtained by connecting the unstable and stable manifolds of the equilibrium points of the asymptotically autonomous equations. Such connections are performed by means of orbit paths of an intermediate equation, which represents the behavior of the system during a suitable interval of transition $\left[t_{-}, t_{+}\right]$between the asymptotic states. Multiple connecting solutions arise when such interval length is sufficiently large. Lower estimates for $t_{+}-t_{-}$will be provided in terms of time mappings, which can be expressed by Abelian integrals. This kind of approach is also reminiscent of some topological methods for the study of Sturm-Liouville boundary value problems. Indeed, a solution that satisfies the Sturm-Liouville boundary conditions can be interpreted as a trajectory in the phase-plane that connects two lines (see [14-17]). Generalized Sturm-Liouville solutions, which connect the graphs of two functions or given planar continua, have been considered as well (see [18-20]). Often these problems can be settled in the framework of the theory of ODEs with nonlinear boundary conditions (see [21, 22]).

In order to make our approach more transparent, we are going to perform our analysis for equation (1.5). This choice is motivated by the sake of avoiding unnecessary technicalities. Our arguments can be modified in a straightforward manner in the case of more general equation (1.1), with $f$ satisfying $(*)$ (see Section 3 ). Homoclinic and heteroclinic orbits can be interpreted as solutions for some boundary value problems on unbounded intervals. In the last section we also outline possible applications of our approach to boundary value problems on a compact interval (like the Sturm-Liouville one).

Besides his manifold achievements in different areas of mathematics, Professor Jean Mawhin is one of the pioneers in the study of topological methods for nonlinear boundary value problems. It is a pleasure and an honor to have the possibility to dedicate our work to his important contributions in this area.

\section{Homoclinic and heteroclinic solutions: multiplicity results}

\subsection{General setting}

We consider the second-order nonlinear differential equation

$$
x^{\prime \prime}+2 k x-2 g(t) x^{3}=0
$$

and its equivalent system in the phase-plane

$$
\left\{\begin{array}{l}
x^{\prime}=y, \\
y^{\prime}=-2 k x+2 g(t) x^{3},
\end{array}\right.
$$


where $k>0$ is a fixed coefficient and $\left.\left.g: \mathbb{R} \rightarrow \mathbb{R}_{0}^{+}:=\right] 0,+\infty\right)$ is a bounded measurable function. Solutions of (2.1) are meant in the generalized (i.e., Carathéodory) sense (see [23]). Actually, in our results a step function $g(t)$ with only two jumps is considered and the solutions are piecewise smooth.

In the particular case of a constant coefficient $g(t) \equiv \mu>0$, an elementary analysis of the system

$$
\left\{\begin{array}{l}
x^{\prime}=y, \\
y^{\prime}=-2 k x+2 \mu x^{3}
\end{array}\right.
$$

shows that the associated phase portrait presents a center $O=(0,0)$ and two saddle points $P_{ \pm}:=\left( \pm(k / \mu)^{\frac{1}{2}}, 0\right)$. These saddles are connected by two heteroclinic solutions (one connecting $P_{-}$to $P_{+}$in the upper half-plane and a symmetric one from $P_{+}$to $P_{-}$in the lower half-plane). The heteroclinic orbits are described by

$$
y^{2}=\mu\left(x^{2}-(k / \mu)\right)^{2} \text { for }-(k / \mu)^{\frac{1}{2}}<x<(k / \mu)^{\frac{1}{2}} .
$$

In [1] Belmonte-Beitia and Torres supposed that the weight function $g(t)$ is even and $T$ periodic and proved the existence of a heteroclinic solution connecting two periodic stationary states. Such a result generalizes to the case of periodic coefficients the situation described above for the autonomous system.

As already observed in the introduction, equation (2.1) has been already studied by various authors for its relevance in many applicative models. The aim of the present paper is to provide multiplicity results for heteroclinic and also homoclinic solutions of equation (2.1). A possible way to obtain this goal is to assume that the weight function $g(t)$ has a different behavior at infinity and in some intermediate time interval. In this setting, a first step consists of analyzing the situation in which $g(t)$ is a stepwise function assuming only two values. We believe that the study of such a simplified case may lead to more general considerations, in which, for instance, $g(t)$ is eventually periodic. In this way, multiplicity results for Belmonte-Beitia and Torres's model can be obtained (see Section 3 for a brief overview concerning possible applications of our approach). Equation (2.1) with a periodic stepwise coefficient $g(t)$ has recently been studied in [24] in the context of chaotic-like dynamics.

As a tool, in our paper, we use the Poincaré map associated to system (2.2). Given a fixed time interval $\left[t_{0}, t_{1}\right]$ and a point $z \in \mathbb{R}^{2}$, we indicate by $\zeta\left(\cdot, t_{0}, z\right)$ the solution $\zeta(t)=$ $(x(t), y(t))$ of (2.2) satisfying the initial condition $\zeta\left(t_{0}\right)=z$. The Poincaré map on $\left[t_{0}, t_{1}\right]$ is defined as

$$
\Phi=\Phi_{t_{0}}^{t_{1}}: z \mapsto \zeta\left(t_{1}, t_{0}, z\right)
$$

From the fundamental theory of differential equations it follows that the domain $D_{\Phi}$ of $\Phi$ is an open subset of the plane and $\Phi$ is an orientation preserving homeomorphism of $D_{\Phi}$ onto its image $\Phi\left(D_{\Phi}\right)$. 


\subsection{Analysis of the equation}

Let us consider system (2.2) for the stepwise weight function $g(t)$, defined as

$$
g(t)= \begin{cases}\mu_{1} & \text { for } t \leq t_{-} \text {or } t \geq t_{+}, \\ \mu_{0} & \text { for } t_{-}<t<t_{+}\end{cases}
$$

where

$$
t_{-}<t_{+} \text {and } 0<\mu_{0}<\mu_{1} \text {. }
$$

System (2.2) can be seen as the superposition of the autonomous systems

$$
\left\{\begin{array}{l}
x^{\prime}=y \\
y^{\prime}=-2 k x+2 \mu_{1} x^{3}
\end{array}\right.
$$

and

$$
\left\{\begin{array}{l}
x^{\prime}=y, \\
y^{\prime}=-2 k x+2 \mu_{0} x^{3} .
\end{array}\right.
$$

The first one describes the asymptotic behavior of the equation. We are going to use the orbits of the second system for connecting unstable/stable manifolds of (2.6) during the time interval $\left[t_{-}, t_{+}\right]$. Figure 1 shows the superposition of the phase portraits of the two systems.

First of all, we briefly analyze the structure of system (2.6). Its equilibrium points are the origin (which is a center) and two saddle points $P_{ \pm}=\left( \pm \xi_{1}, 0\right)$, where

$$
\xi_{1}:=\left(k / \mu_{1}\right)^{\frac{1}{2}}
$$

The stable and unstable manifolds of $P_{ \pm}$are illustrated in Figure 2. The sets $\ell\left(P_{-}\right) \cup$ $\mathcal{L}\left(P_{+}, P_{-}\right)$and $\mathcal{O}\left(P_{-}\right) \cup \mathcal{L}\left(P_{-}, P_{+}\right)$are, respectively, the stable and the unstable manifolds of $P_{-}$. Symmetrically, $\ell\left(P_{+}\right) \cup \mathcal{L}\left(P_{-}, P_{+}\right)$and $\mathcal{O}\left(P_{+}\right) \cup \mathcal{L}\left(P_{+}, P_{-}\right)$are the stable and the unstable manifolds of $P_{+}$.

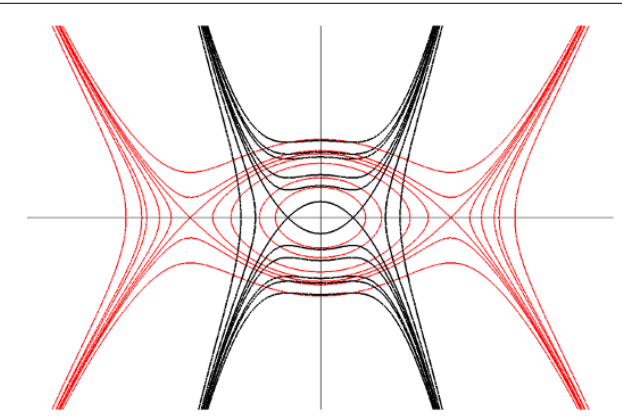

Figure 1 In the present figure we have considered the superposition of the phase portraits of systems (2.6) (in darker color) and (2.7) (in lighter color) with $k=1, \mu_{1}=4, \mu_{0}=1 / 4$. For graphical reasons, a slightly different $x$ - and $y$-scaling has been used. 


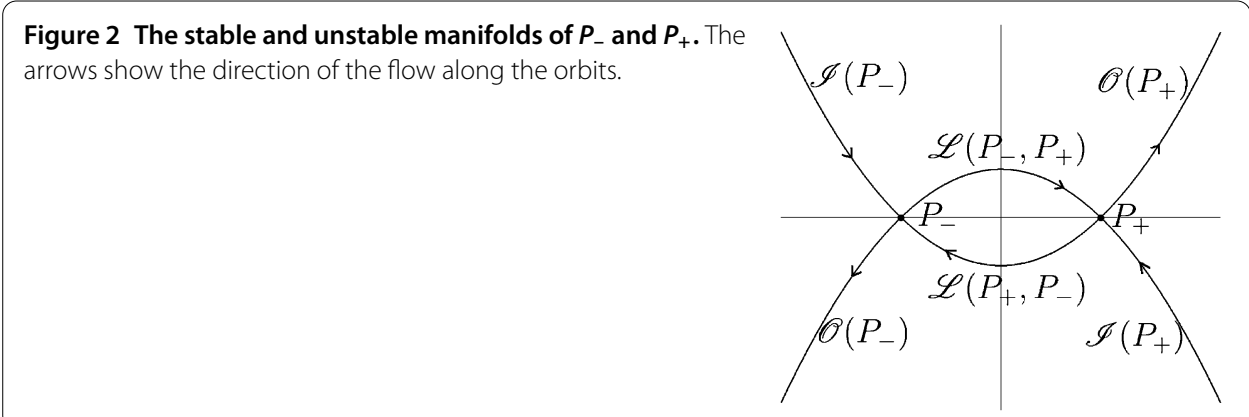

In the case of the cubic nonlinearity, we find

$$
\begin{aligned}
& \ell\left(P_{-}\right)=\left\{(x, y): y=\left|\mu_{1} x^{2}-k\right| / \sqrt{\mu_{1}}, x<-\left(k / \mu_{1}\right)^{\frac{1}{2}}\right\}, \\
& \mathcal{O}\left(P_{-}\right)=\left\{(x, y): y=-\left|\mu_{1} x^{2}-k\right| / \sqrt{\mu_{1}}, x<-\left(k / \mu_{1}\right)^{\frac{1}{2}}\right\}, \\
& \ell\left(P_{+}\right)=\left\{(x, y): y=-\left|\mu_{1} x^{2}-k\right| / \sqrt{\mu_{1}}, x>\left(k / \mu_{1}\right)^{\frac{1}{2}}\right\}, \\
& \mathcal{O}\left(P_{+}\right)=\left\{(x, y): y=\left|\mu_{1} x^{2}-k\right| / \sqrt{\mu_{1}}, x>\left(k / \mu_{1}\right)^{\frac{1}{2}}\right\}, \\
& \mathcal{L}\left(P_{-}, P_{+}\right)=\left\{(x, y): y=\left|\mu_{1} x^{2}-k\right| / \sqrt{\mu_{1}},-\left(k / \mu_{1}\right)^{\frac{1}{2}}<x<\left(k / \mu_{1}\right)^{\frac{1}{2}}\right\}, \\
& \mathcal{L}\left(P_{+}, P_{-}\right)=\left\{(x, y): y=-\left|\mu_{1} x^{2}-k\right| / \sqrt{\mu_{1}},-\left(k / \mu_{1}\right)^{\frac{1}{2}}<x<\left(k / \mu_{1}\right)^{\frac{1}{2}}\right\} .
\end{aligned}
$$

The solutions of system (2.2) with $g(t)$ defined as in (2.5) will be obtained by connecting suitably chosen parts of stable and unstable manifolds of (2.6) with trajectories of (2.7). More in detail, we proceed as follows.

Due to the Hamiltonian nature of the equation under consideration, we can consider the 'energy' level lines of system (2.7), given by

$$
E(x, y):=y^{2}+2 k x^{2}-\mu_{0} x^{4}=c .
$$

We also set

$$
\Gamma^{c}:=\left\{(x, y):-\xi_{0} \leq x \leq \xi_{0}, E(x, y)=c\right\}
$$

where

$$
\xi_{0}:=\left(k / \mu_{0}\right)^{\frac{1}{2}}
$$

The curve $\Gamma^{c}$ is the part of the line at level $c \in \mathbb{R}$ in the strip $\left[-\xi_{0}, \xi_{0}\right] \times \mathbb{R}$. For every $c$ such that

$$
0<c<\ell_{0}:=\frac{k^{2}}{\mu_{0}}
$$

the set $\Gamma^{c}$ is a periodic orbit of (2.7) run in the clockwise sense, which intersects the $y$-axis at the points $(0, \pm \sqrt{c})$. The (minimal) period $\tau_{c}$ of $\Gamma^{c}$ can be expressed by the following 
time-mapping formula:

$$
\tau_{c}=2 \int_{s_{0}}^{s_{1}} \frac{d x}{\sqrt{c-2 F(x)}}
$$

where $F(x)$ is the potential associated to the equation and $s_{0}, s_{1}$ are such that

$$
F\left(s_{0}\right)=F\left(s_{1}\right)=c / 2 \quad \text { with }-\xi_{0}<s_{0}<0<s_{1}<\xi_{0} .
$$

In our case,

$$
F(x)=k x^{2}-\frac{\mu_{0}}{2} x^{4}
$$

and by symmetry $s_{0}=-s_{1}$. More in detail, we can express the period $\tau_{c}$ by means of an elliptic integral in the following way:

$$
\tau_{c}=\frac{4}{\mu_{0}} \int_{0}^{s_{1}} \frac{d x}{\sqrt{\left(a^{2}-x^{2}\right)\left(b^{2}-x^{2}\right)}},
$$

for $a:=s_{1}, b:=\left(\frac{2 k^{2}}{\mu_{0}^{2}}-s_{1}^{2}\right)^{1 / 2}$ and $0<s_{1}<\xi_{0}, 0<c=2 k s_{1}^{2}-\mu_{0} s_{1}^{4}<\ell_{0}$. Notice that $0<a<b$ in (2.10). Therefore

$$
\tau_{c}=\frac{4}{b \mu_{0}} \operatorname{sn}^{-1}(1, a / b)
$$

where $\operatorname{sn}(\cdot, k)$ is the Jacobi elliptic sine function of modulus $k:=a / b$ (see $[25,26])$.

The level line $\Gamma^{\ell_{0}}$ contains the saddle points $Q_{ \pm}:=\left( \pm \xi_{0}, 0\right)$ of system $(2.7)$ and their heteroclinic connections $\mathscr{L}\left(Q_{-}, Q_{+}\right)$in the upper half-plane and $\mathscr{L}\left(Q_{+}, Q_{-}\right)$in the lower half-plane. The energy level

$$
\ell_{1}:=\frac{k^{2}}{\mu_{1}}
$$

corresponds to the closed curve $\Gamma^{\ell_{1}}$, which is tangent to $\mathscr{L}\left(P_{-}, P_{+}\right)$and $\mathcal{L}\left(P_{+}, P_{-}\right)$. Moreover, for

$$
\ell^{*}:=\frac{k^{2}}{\mu_{1}}\left(2-\frac{\mu_{0}}{\mu_{1}}\right)
$$

the energy level line $\Gamma^{\ell^{*}}$ contains the saddle points $P_{ \pm}$of system (2.6).

Using the parameters $\ell_{0}, \ell_{1}$ and $\ell^{*}$, we define the regions

$$
\begin{aligned}
& \mathcal{E}:=\left\{(x, y):-\xi_{0} \leq x \leq \xi_{0}, \ell_{1} \leq E(x, y) \leq \ell^{*}\right\}, \\
& \mathcal{F}:=\left\{(x, y):-\xi_{0} \leq x \leq \xi_{0}, \ell^{*}<E(x, y)<\ell_{0}\right\} .
\end{aligned}
$$

Both $\mathscr{E}$ and $\mathcal{F}$ are invariant sets for (2.7); they are filled by periodic orbits. In the sequel, the orbits in the region $\mathcal{E}$ will be called internal, while those in $\mathcal{F}$ will be called external. 


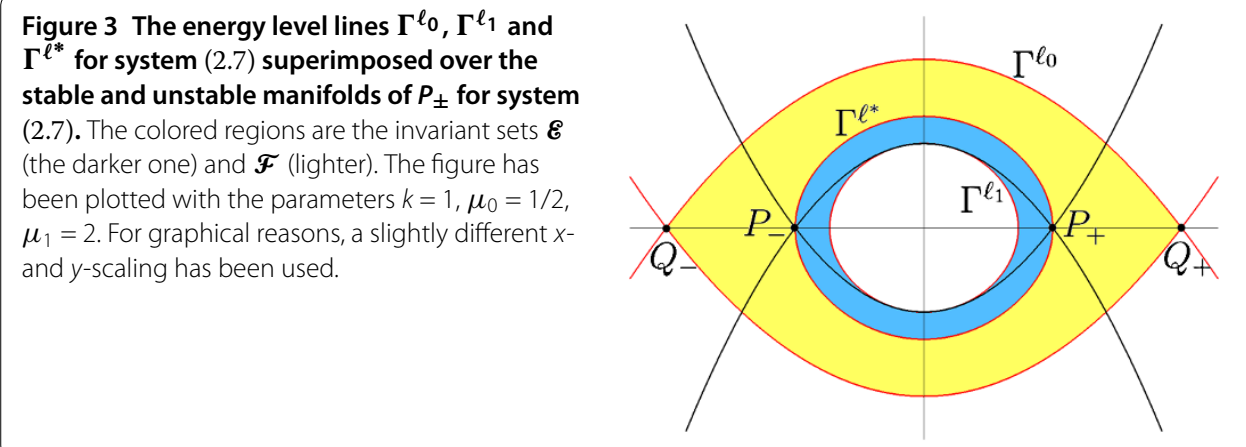

The choice of these names is made in order to distinguish the trajectories with respect to $\Gamma^{\ell^{*}}$, which contains the saddle points $P_{ \pm}$of system (2.6). (See Figure 3.)

In order to obtain homoclinic or heteroclinic solutions for system (2.2) with $g(t)$ defined as in (2.5), we connect the unstable manifold of the point $A \in\left\{P_{-}, P_{+}\right\}$to the stable manifold of $B \in\left\{P_{-}, P_{+}\right\}$via an orbit path of $\Gamma^{c}$ for $c \in\left[\ell_{1}, \ell_{0}\right.$ [. Actually, this is the only way to get the desired solutions. If $c \leq \ell^{*}$, such connection will follow a trajectory $(x(t), y(t))$ with $-\xi_{0} \leq x(t) \leq \xi_{0}$, so it will lie in the invariant annular region $\mathcal{E}$ bounded by $\Gamma^{\ell_{1}}$ and $\Gamma^{\ell^{*}}$. On the contrary, for $c>\ell^{*}$ the connection lies in the set $\mathcal{F}$ between $\Gamma^{\ell^{*}}$ and $\Gamma^{\ell_{0}}$.

To provide more explicit details, we consider the case $A=P_{+}$. A solution of (2.2), which is homoclinic to $P_{+}$, can be produced in two ways. One consists in connecting $\mathcal{O}\left(P_{+}\right)$to $\ell\left(P_{+}\right)$, using an orbit path of $\Gamma^{c}$ for $\left.c \in\right] \ell^{*}, \ell_{0}$ [. Another possibility is given by connecting a point of $\mathscr{L}\left(P_{+}, P_{-}\right)$to a point of $\mathcal{L}\left(P_{-}, P_{+}\right)$via an orbit path of $\Gamma^{c}$ for $c \in\left[\ell_{1}, \ell^{*}\left[\right.\right.$. For $c=\ell^{*}$, we can obtain our solution if and only if $t_{+}-t_{-}$is an integer multiple of the fundamental period $\tau_{\ell^{*}}$ of the orbit $\Gamma^{\ell^{*}}$. Indeed, such a solution will be constantly equal to $P_{+}$for $\left.t \notin\right] t_{-}, t_{+}[$, and for $t \in\left[t_{-}, t_{+}\right]$it coincides with the periodic solution of (2.7) such that $x\left(t_{-}\right)=\xi_{1}$ and $y\left(t_{-}\right)=0$. This solution makes $\left(t_{+}-t_{-}\right) / \tau_{\ell^{*}}$ turns around the origin for $t \in\left[t_{-}, t_{+}\right]$.

Similar considerations can be developed with respect to heteroclinic solutions. For instance, a heteroclinic orbit from $P_{+}$to $P_{-}$can be obtained as follows: by connecting a point of $\mathcal{O}\left(P_{+}\right)$with one of $\ell\left(P_{-}\right)$using an orbit path of $\Gamma^{c}$ for $\left.c \in\right] \ell^{*}, \ell_{0}$ [, or by a connection of two different points of $\mathscr{L}\left(P_{+}, P_{-}\right)$via an orbit path of $\Gamma^{c}$ for $c \in\left[\ell_{1}, \ell^{*}\right.$ [. For $c=\ell^{*}$, we can obtain our solution if and only if $t_{+}-t_{-}$is an odd multiple of $\tau_{\ell^{*}} / 2$, where $\tau_{\ell^{*}}$ is the period of the orbit $\Gamma^{\ell^{*}}$. Indeed, such a solution will be constantly equal to $P_{+}$for $t \in\left(-\infty, t_{-}\right]$and constantly equal to $P_{-}$for $t \in\left[t_{+},+\infty\right)$. Moreover, it coincides for $t \in\left[t_{-}, t_{+}\right]$with the solution of (2.7) such that $x\left(t_{-}\right)=\xi_{1}$ and $y\left(t_{-}\right)=0$. This solution makes $2\left(t_{+}-t_{-}\right) / \tau_{\ell^{*}}$ half-turns around the origin for $t \in\left[t_{-}, t_{+}\right]$.

Figure 4, although not exhaustive of all the conceivable cases, summarizes several different possibilities. Indeed, a trajectory homoclinic to $P_{+}$can be obtained as follows: move by system (2.6) along $\mathcal{O}\left(P_{+}\right)$from $P_{+}$to the intersection point of $\mathcal{O}\left(P_{+}\right)$with a closed curve external to $P_{+}$, namely $\delta$ (for $t \in\left(-\infty, t_{-}\right]$). This point is put in evidence with a small black circle. Next, follow $\delta$ (by system (2.7)) in order to reach the intersection of $\delta$ with $\ell\left(P_{+}\right)$ (for $t \in\left[t_{-}, t_{+}\right]$). Such an intersection point is indicated by a grey square. Finally, switch to system (2.6) and move toward $P_{+}$along $\ell\left(P_{+}\right)($for $t \in[t+,+\infty)$ ). With a similar procedure, we can obtain a heteroclinic trajectory from $P_{+}$to $P_{-}$. In fact, we can move by system (2.6) along $\mathcal{O}\left(P_{+}\right)$from $P_{+}$to the intersection point of $\mathcal{O}\left(P_{+}\right)$with a closed curve external to $P_{+}$, namely $\gamma$ (for $t \in\left(-\infty, t_{-}\right]$). Next, follow $\gamma$ (by system (2.7)) to the intersection with 
Figure 4 In the present figure we have considered the superposition of (2.6) and (2.7) for $k=1, \mu_{1}=4, \mu_{0}=1 / 4$. The closed curves $\alpha, \beta$, $\gamma, \delta$ represent four different level lines of system (2.7): the curves $\alpha$ and $\beta$ in $\boldsymbol{E}$ correspond to a level $c \in] \ell_{1}, \ell^{*}[$, while $\gamma$ and $\delta$ in $\mathscr{F}$ refer to $c \in] \ell^{*}, \ell_{0}[$. In order to describe a connection from an unstable manifold to a stable one of system (2.6) via an orbit path of (2.7), we have marked with a black circle possible starting points and with a grey square some available end points on the same level lines.

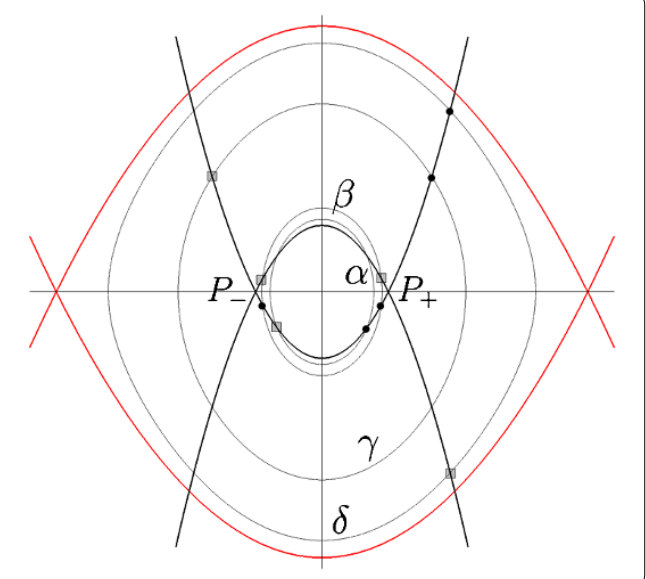

$\ell\left(P_{-}\right)$(for $\left.t \in\left[t_{-}, t_{+}\right]\right)$. Finally, switch to system (2.6) and move toward $P_{-}$along $\ell\left(P_{-}\right)$(for $\left.t \in\left[t_{+},+\infty\right)\right)$.

In general, the connections through the trajectories of system (2.7) will either involve only an arc of the closed curve $\Gamma^{c}$, or they will require to perform a certain number of winds around the origin, depending on the available time. If the length of the time interval $\left[t_{-}, t_{+}\right]$is small, only the first case is possible. However, if $t_{+}-t_{-}$is large enough, more choices arise. For instance, looking at the orbit $\delta$ in Figure 4 , we could make a certain number of loops from the black circle before reaching the grey square on the same orbit. Similar considerations apply to the heteroclinic trajectory described above with reference to $\gamma$.

Until now we have described the 'external connections', namely those which lie in the region $\mathcal{F}$. Further possibilities appear if we consider 'internal connections' by means of orbit paths contained in $\mathcal{E}$. For example, if we look at the orbit $\beta$ in Figure 4 , we can obtain trajectories homoclinic to $P_{+}$by choosing one of the two black circles as a starting point from $\mathcal{L}\left(P_{+}, P_{-}\right)$and one of the two grey squares as an end point on $\mathcal{L}\left(P_{-}, P_{+}\right)$. Loops along $\beta$ will be permitted if the time is sufficiently large. The construction of heteroclinic orbits from $P_{+}$to $P_{-}$via the line $\alpha$ follows a similar procedure.

\subsection{Study of the Poincaré map}

Another possible point of view to describe the previous construction of homoclinic and heteroclinic solutions consists in considering the Poincaré map associated to system (2.7) for the time interval $\left[t_{-}, t_{+}\right]$. Due to the autonomous nature of this system, we have that $\Phi_{t_{-}}^{t_{+}}=\Phi_{0}^{t_{+}-t_{-}}$, denoted simply by $\Phi$ when no confusion may occur. Moreover, the region

$$
\mathcal{W}:=\left\{(x, y):-\xi_{0} \leq x \leq \xi_{0}, E(x, y) \leq \ell_{0}\right\}
$$

is a compact invariant set contained in the domain of $\Phi$. Let us denote by $y:=\overline{\mathcal{O}\left(P_{+}\right)} \cap \overline{\mathcal{F}}$ the part of the unstable manifold $\mathcal{O}\left(P_{+}\right)$between $P_{+}$and $\Gamma^{\ell_{0}}$, including the extreme points. Hence the external connections can be precisely described by looking at the intersection points of $\Phi(\mathcal{Y})$ with $\ell\left(P_{+}\right)$(for the homoclinics) and with $\ell\left(P_{-}\right)$(for the heteroclinics), as illustrated in Figure 5.

With reference to Figure 5, we observe that the intersection point of $\mathcal{O}\left(P_{+}\right)$with $\Gamma^{\ell_{0}}$ (indicated by a small circle) is moved by $\Phi$ along $\Gamma^{\ell_{0}}$ to a point very close to $Q_{+}$. On 
Figure 5 In the present figure we show the transformation of $\mathcal{y}$ under the Poincaré map $\Phi$ for $\boldsymbol{t}_{+}-\boldsymbol{t}_{-}=\mathbf{5 . 5}$. The parameters used are $k=1, \boldsymbol{\mu}_{1}$ $=2, \mu_{0}=1 / 2$. For graphical reasons a slightly different $x$ - and $y$-scaling has been used.

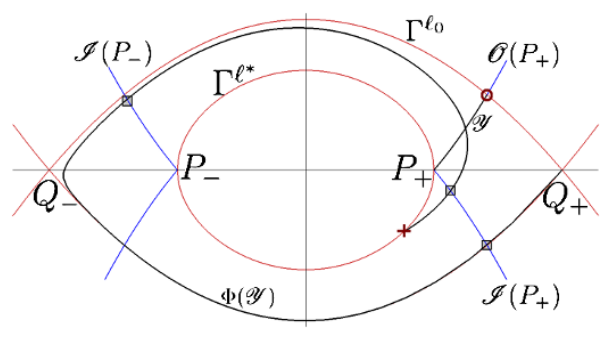

Figure 6 In the present figure we show the transformation of $\mathcal{L}:=\overline{\mathcal{L}\left(P_{+}, P_{-}\right)}$under the Poincaré map $\Phi$ for $t_{+}-t_{-}=5.5$, the same time considered in Figure 5. The parameters used are $k$ $=1, \mu_{1}=2, \mu_{0}=1 / 2$, as before. The point indicated by a cross is precisely the same point $\Phi\left(P_{+}\right)$of Figure 5. Notice that for this time interval the point makes a little more than a complete turn around the origin. For graphical reasons, a slightly different $x$ and $y$-scaling has been used.

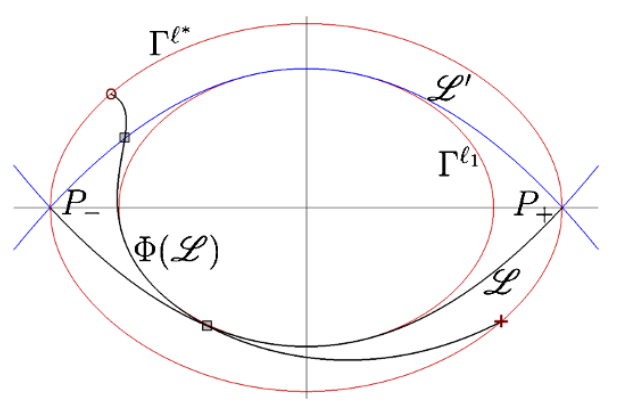

the other hand, during the time interval $\left[t_{-}, t_{+}\right]$, the point $P_{+}$makes a little more than a complete turn around the origin; the final position $\Phi\left(P_{+}\right)$is indicated by a cross. The $\operatorname{arc} \Phi(y)$ is a spiral-like curve with one end near $Q_{+}$and the other on $\Gamma^{\ell^{*}}$. For the time length $t_{+}-t_{-}=5.5$, considered in Figure 5, there are precisely two intersections of $\Phi(\mathcal{y})$ with $\ell\left(P_{+}\right)$, corresponding to external homoclinic solutions. Meanwhile, we have also an external heteroclinic solution due to the intersection of $\Phi(\mathcal{Y})$ with $\ell\left(P_{-}\right)$. All these three intersection points have been indicated by a grey square (following the same convention used in Figure 4).

In order to describe further these solutions, let us consider their energy at the time $t_{+}$ that we denote by $k_{1}, k_{2}, k_{3}$, with $\ell^{*}<k_{3}<k_{2}<k_{1}<\ell_{0}$. Let us define the points $H_{i}$ as $\left\{H_{i}\right\}=\mathcal{O}\left(P_{+}\right) \cap \Gamma^{k_{i}}$ for $i=1,2,3$. The solution $\zeta(t)=(x(t), y(t))$ of $(2.2)$ with $\zeta\left(t_{-}\right)=H_{1}$ or $\zeta\left(t_{-}\right)=H_{3}$ has the following behavior: $\zeta(t) \rightarrow P_{+}$for $t \rightarrow \pm \infty$, with $x(t)>\xi_{1}$ and convex for $t \notin] t_{-}, t_{+}$. Moreover, $x(t)$ is increasing on $\left(-\infty, t_{-}\right]$and decreasing on $\left[t_{+},+\infty\right)$. On the interval $\left[t_{-}, t_{+}\right]$, if $i=1$, the solution $x(t)$ is concave, while if $i=3$, the solution has two maxima and one (negative) minimum separated by two simple zeros. The solution of system (2.2) with $\zeta\left(t_{-}\right)=H_{2}$ is such that $\zeta(t) \rightarrow P_{+}$for $t \rightarrow-\infty$ with $x(t)>\xi_{1}$ and $\zeta(t) \rightarrow P_{-}$ for $t \rightarrow+\infty$ with $x(t)<-\xi_{1} ; x(t)$ is convex for $\left.t \notin\right] t_{-}, t_{+}$[. Moreover, the solution has one maximum and one minimum separated by one simple zero.

Even if the time interval length $t_{+}-t_{-}$is small, we always have at least one intersection of $\Phi(y)$ with $\ell\left(P_{+}\right)$and thus a homoclinic solution. On the other hand, if $t_{+}-t_{-}$ grows, the spiral curve $\Phi(\mathcal{y})$ will wind more times around the origin, hence more homoclinic/heteroclinic solutions will appear.

If we look for the internal connections (made by orbit paths lying in the region $\varepsilon$ ), we proceed as follows.

Let us set $\mathcal{L}:=\overline{\mathcal{L}\left(P_{+}, P_{-}\right)}$and denote by $\mathcal{L}^{\prime}:=\overline{\mathcal{L}\left(P_{-}, P_{+}\right)}$its symmetric part with respect to the $x$-axis. Figure 6 illustrates the involved geometry from the point of view of the Poincaré map. We observe that all the points of $\mathcal{L}$ are contained in $\mathcal{E}$, hence they are periodic points 
(of different periods). As before, during the time interval $\left[t_{-}, t_{+}\right]$, the point $P_{+}$moves clockwise around the origin along $\Gamma^{\ell^{*}}$; the final position $\Phi\left(P_{+}\right) \in \Gamma^{\ell^{*}}$ is indicated by a cross. Symmetrically, the point $P_{-}$performs exactly the same angle around the origin, reaching the final position $\Phi\left(P_{-}\right) \in \Gamma^{\ell^{*}}$, indicated by a small circle. All the other points of $\mathcal{L}\left(P_{+}, P_{-}\right)$ move, under the action of $\Phi$, on the energy level lines $\Gamma^{c}$ with $\ell_{1} \leq c<\ell^{*}$. The arc $\Phi(\mathcal{L})$ is a curve contained in $\mathcal{E}$, connecting the two antipodal points $\Phi\left(P_{ \pm}\right) \in \Gamma^{\ell^{*}}$ and leaning on one point of $\Gamma^{\ell_{1}}$. This tangent point is the image through $\Phi$ of the point $R:=\left(0,-\sqrt{\ell_{1}}\right)$. Such a property of $\Phi(\mathcal{L})$ implies that $\Phi(\mathcal{L})$ intersects both $\mathcal{L}$ and $\mathcal{L}^{\prime}$ (at different points). These two points have been indicated by a grey square in the figure. Accordingly, we always find at least an internal homoclinic solution and an internal heteroclinic one.

Summarizing the above information, we conclude that, for every time interval $\left[t_{-}, t_{+}\right]$, we have at least two solutions which are homoclinic to $P_{+}$(one external and one internal) and one internal heteroclinic solution from $P_{+}$to $P_{-}$.

For the external connections, if the time interval length $t_{+}-t_{-}$grows, the situation can be summarized as follows. The number of external homoclinic/heteroclinic solutions increases, depending on the number of winds around the origin of the curve $\Phi(y)$. Indeed, the end point of such a curve on $\Gamma^{\ell_{0}}$ cannot go beyond $Q_{+}$, while the other end point $\Phi\left(P_{+}\right)$ is free to move on the periodic orbit $\Gamma^{\ell^{*}}$. If we denote by $\eta_{\text {ext }}$ and $v_{\text {ext }}$ the number of external homoclinic and heteroclinic solutions respectively, it holds that $0 \leq \eta_{\text {ext }}-v_{\text {ext }} \leq 1$. From the above discussion, we can conclude that if, for some nonnegative integer $n$, we have $t_{+}-t_{-}>n \tau_{\ell^{*}}$ (where $\tau_{\ell^{*}}$ is the period of $\Gamma^{\ell^{*}}$ ), then $\eta_{\text {ext }} \geq n+1$. An analogous lower bound can be provided for $v_{\text {ext }}$. For a formal proof, see Theorem 2.1.

When $t_{+}-t_{-}$grows, the situation for the internal homoclinic/heteroclinic solutions is more intriguing, as illustrated in Figure 7 and Figure 8. A twist effect depending on the different periods of $\Gamma^{\ell_{1}}$ and $\Gamma^{\ell^{*}}$ produces a double spiral-like curve $\Phi(\mathcal{L})$ when $t_{+}-t_{-}$is sufficiently large.

The fact that $\Phi(\mathcal{L})$ looks like a double spiral depends on the different velocities of the points $P_{ \pm}$and $R=\left(0,-\sqrt{\ell_{1}}\right)$ (which is the intersection of $\mathcal{L}$ with the negative $y$-axis). In fact, since $\tau_{\ell_{1}}<\tau_{\ell^{*}}$, the points on $\Gamma^{\ell_{1}}$ move faster than those of $\Gamma^{\ell^{*}}$. By construction, $P_{ \pm} \in \Gamma^{\ell^{*}}$, while $R \in \Gamma^{\ell_{1}}$. Therefore, when the time gap $t_{+}-t_{-}$is sufficiently large, the number of turns of $\Phi(R)$ around the origin will exceed the number of turns of $\Phi\left(P_{ \pm}\right)$. Accordingly, the image through $\Phi$ of the right part of $\mathcal{L}$ (the sub-arc of $\mathcal{L}$ connecting $P_{+}$to $R$ ) is a spiral that winds a certain number of times in the clockwise sense around the origin.

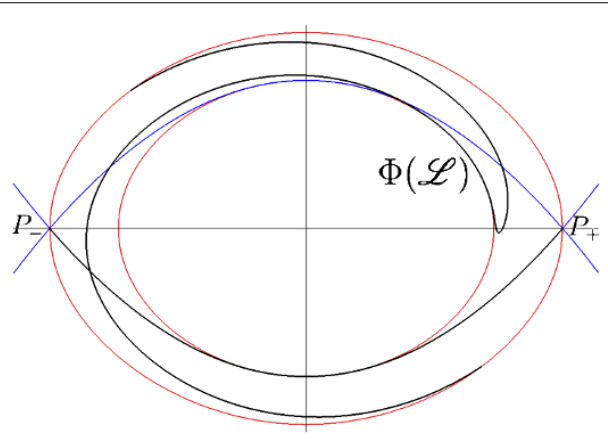

Figure 7 In the present figure we show the transformation of $\mathscr{L}$ under the Poincaré map $\Phi$ for $\boldsymbol{t}_{+}-\boldsymbol{t}_{-}=\mathbf{5 0}$. The parameters used are $k=1, \mu_{1}=2, \mu_{0}=1 / 2$, as before. For graphical reasons, a slightly different $x$ - and $y$-scaling has been used. 
Figure 8 The present figure shows the evolution of $\mathcal{L}$ under the Poincaré map $\Phi$ for time intervals of different lengths. The parameters used are $k=1, \mu_{1}=2, \mu_{0}=1 / 2$, as before, while for $t_{+}-t_{-}$, we have considered the cases $35,60,100$, 150 (from the left to the right). For graphical reasons, a slightly different $x$ - and $y$-scaling has been used.
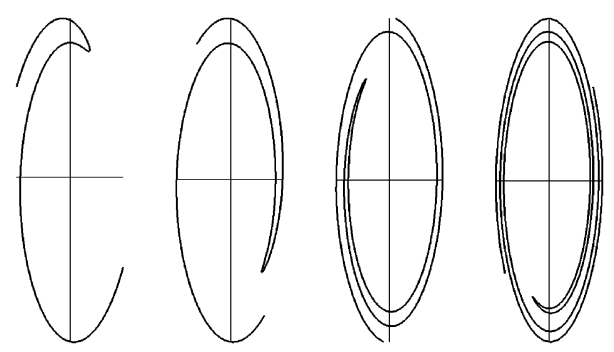

Figure 9 The present figure explains the twist effect due to the different periods of $\Gamma^{\ell_{1}}$ and $\Gamma^{\ell^{*}}$. We have considered the evolution of the part of $\mathcal{L}$ with $x \geq 0$ connecting $P_{+}$to the point $R$ for two different time gaps: $t_{+}-t_{-}=5.5$ and $t_{+}-t_{-}=50$. The corresponding two images $\Phi\left(P_{+}\right)$are indicated by a cross, while the images $\Phi(R)$ are marked by a black dot. The parameters used are $k=1, \mu_{1}=2$, $\mu_{0}=1 / 2$, as before. Notice that the two images $\Phi\left(P_{+}\right)$, although close to each other in the figure, have made a different number of turns around the origin. For graphical reasons, a slightly different $x$ -

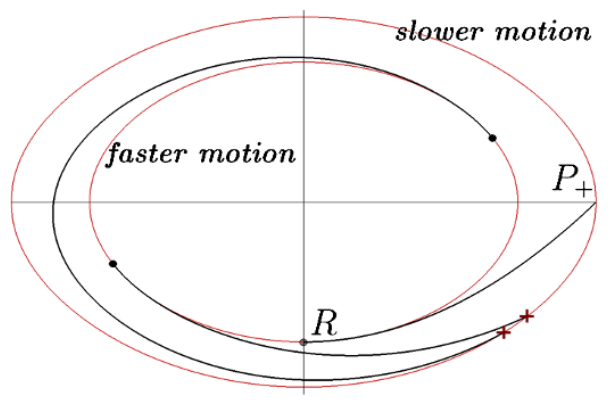
and $y$-scaling has been used.

Indeed, it connects the slower point $\Phi\left(P_{+}\right)$to the faster one $\Phi(R)$ inside the region $\mathcal{E}$, as illustrated in Figure 9. The number of half-turns of this spiral depends on a 'rotational gap' that, for our purposes, it will be convenient to define as

$$
w\left(t_{+}-t_{-}\right):=\left\lceil\frac{2\left(t_{+}-t_{-}\right)}{\tau_{\ell_{1}}}\right\rceil-\left\lfloor\frac{2\left(t_{+}-t_{-}\right)}{\tau_{\ell^{*}}}\right\rfloor-2 .
$$

Similarly, the image through $\Phi$ of the left part of $\mathcal{L}$ (the sub-arc of $\mathcal{L}$ connecting $R$ to $P_{-}$) is a spiral-like curve winding a certain number of times in the counterclockwise sense around the origin. Indeed, it connects the points $\Phi(R)$ and $\Phi\left(P_{-}\right)$inside the region $\varepsilon$ (the first point moves at a faster speed than the second one). Again, the number of half-turns of this second curve will depend on $w\left(t_{+}-t_{-}\right)$. In the end, gluing together the two spirallike curves, we conclude that, if $t_{+}-t_{-}$is sufficiently large, the arc $\Phi(\mathcal{L})$ will appear like a double spiral with a central 'hook'. To better describe the structure of $\Phi(\mathcal{L})$, we should observe that the 'tip of the hook' is not $\Phi(R)$, but it is the image through $\Phi$ of a point (close to $R$ ) on the left part of $\mathcal{L} \backslash\{R\}$ (we owe this remark to the referee). For a formal proof based on the argument outlined above, see Theorem 2.2.

In preparation for this theorem, we introduce the following notation. Given a positive real number $S$ and an energy level $\ell \in\left[\ell_{1}, \ell^{*}\right]$, we denote by $\underline{n}(S, \ell):=\left\lfloor\frac{2 S}{\tau_{\ell}}\right\rfloor$ the lower integer part of $S /\left(\tau_{\ell} / 2\right)$, and by $\bar{n}(S, \ell):=\left\lceil\frac{2 S}{\tau_{\ell}}\right\rceil$ the upper integer part of $S /\left(\tau_{\ell} / 2\right)$. By definition, during a time interval of length $S$, a point on the orbit $\Gamma^{\ell}$ makes more that $\bar{n}(S, \ell)-1$ halfturns around the origin, but less that $\underline{n}(S, \ell)+1$ half-turns. Thus the rotational gap defined above can be written as $w(T)=\bar{n}\left(T, \ell_{1}\right)-\underline{n}\left(T, \ell^{*}\right)-2$ for $T=t_{+}-t_{-}$. By definition, in the open interval $] 2 T / \tau_{\ell^{*}}, 2 T / \tau_{\ell_{1}}$, the smallest integer is $\underline{n}\left(T, \ell^{*}\right)+1$, while the largest integer is $\bar{n}\left(T, \ell^{*}\right)-1$. Hence, the open interval $] 2 T / \tau_{\ell^{*}}, 2 T / \tau_{\ell_{1}}$ [ contains $w(T)+1$ positive integers. 


\subsection{Conclusion}

After the preliminary study of the previous subsections, we are now in a position to express our results for the equation

$$
x^{\prime \prime}+2 k x-2 g(t) x^{3}=0
$$

as statements with a formal proof. Recall that the weight function $g(t)$ is defined as follows:

$$
g(t)= \begin{cases}\mu_{1} & \text { for } t \leq t_{-} \text {or } t \geq t_{+} \\ \mu_{0} & \text { for } t_{-}<t<t_{+}\end{cases}
$$

where

$$
t_{-}<t_{+} \text {and } 0<\mu_{0}<\mu_{1} \text {. }
$$

We also set $T:=t_{+}-t_{-}$and consider the Poincaré map $\Phi_{0}^{T}$ associated to the system

$$
\left\{\begin{array}{l}
x^{\prime}=y, \\
y^{\prime}=-2 k x+2 \mu_{0} x^{3} .
\end{array}\right.
$$

In order to make the exposition more clear, we have decided to consider separately the cases of external and internal connections.

Theorem 2.1 (External connections) Under the above assumptions, the following results hold for solutions which satisfy the energy condition

$$
\left.x^{\prime}(t)^{2}+2 k x(t)^{2}-\mu_{0} x(t)^{4} \in\right] \ell^{*}, \ell_{0}[, \quad \forall t \in \mathbb{R}:
$$

- there always exist a (positive) solution homoclinic to $P_{+}$and a (negative) solution homoclinic to $P_{-}$;

- if $t_{+}-t_{-}>\tau_{\ell^{*}} / 2$, there always exist a heteroclinic solution from $P_{+}$to $P_{-}$and a heteroclinic solution from $P_{-}$to $P_{+}$, both with exactly one zero;

- if $t_{+}-t_{-}>n \tau_{\ell^{*}}$ (for some integer $n \geq 1$ ) then, for each integer $j$ with $1 \leq j \leq n$, there exist at least one solution homoclinic to $P_{+}$and one solution homoclinic to $P_{-}$, both with exactly $2 j$ zeros;

- if $t_{+}-t_{-}>(n+1) \tau_{\ell^{*}} / 2$ (for some integer $\left.n \geq 1\right)$ then, for each integer $j$ with $1 \leq j \leq n$, there exist at least one heteroclinic solution from $P_{+}$to $P_{-}$and one from $P_{-}$to $P_{+}$, both with exactly $2 j+1$ zeros.

Proof As a first step, we focus our attention on the search of solutions homoclinic to the point $P_{+}$. The case of homoclinics to $P_{-}$is analogous, thus it will be omitted.

Recalling that $T=t_{+}-t_{-}$, our goal is to find points on $\mathcal{O}\left(P_{+}\right)$which are moved by the Poincaré map $\Phi_{0}^{T}$ on $\ell\left(P_{+}\right)$.

We introduce in the phase-plane for (2.7), a system of polar coordinates $(\theta, \rho)$, with center in the origin. The initial points in the arc $y$ (which is the closure of the intersection of $\mathcal{O}\left(P_{+}\right)$with the external region $\left.\mathcal{F}\right)$ are parameterized as $\gamma(s):=(\rho(s) \cos \theta(s), \rho(s) \sin \theta(s))$, 
for $s \in[0,1]$, with $\gamma(0)=P_{+}, \gamma(1) \in \Gamma^{\ell_{0}}$ and $E(\gamma(s))$ strictly increasing with $s$. The target set is the symmetric of $y$ with respect to the $x$-axis. It will be denoted by $y^{\prime}$ and parameterized by reversing the angle $\theta(s)$.

Using the same polar coordinates to represent the solutions of (2.7), we can express the final points $\Phi_{0}^{T}(\gamma(s))$ as

$$
\Phi_{0}^{T}(\gamma(s))=(\rho(T, s) \cos \theta(T, s), \rho(T, s) \sin \theta(T, s))
$$

with

$$
\rho(0, s)=\rho(s), \quad \theta(0, s)=\theta(s) .
$$

We notice that

$$
(x(t), y(t))=(\rho(t, s) \cos \theta(t, s), \rho(t, s) \sin \theta(t, s))
$$

is the solution of (2.7) (at the time $t$ ), which departed from the point $\gamma(s)$ at the time $t=0$. Observe that, for every $s \in[0,1]$, the map

$$
t \mapsto \theta(t, s)
$$

is strictly decreasing on $[0,+\infty)$ (this is an equivalent way to express the fact that the solutions turn around the origin in the clockwise sense).

With these positions, we obtain an external solution homoclinic to $P_{+}$if and only if there exists $\hat{s} \in] 0,1\left[\right.$ such that $\Phi_{0}^{T}(\gamma(\hat{s})) \in \mathcal{Y}^{\prime}$. This happens if and only if

$$
\rho(T, \hat{s})=\rho(\hat{s}) \quad \text { and } \quad \theta(T, \hat{s})=-\theta(\hat{s})-2 j \pi,
$$

for some nonnegative integer $j$. In this case, if we denote by $\hat{x}(\cdot)$ the corresponding homoclinic solution of (2.1) such that $\left(\hat{x}\left(t_{-}\right), \hat{x}^{\prime}\left(t_{-}\right)\right)=\gamma(\hat{s})$, we have that $\left.E\left(\hat{x}(t), \hat{x}^{\prime}(t)\right) \in\right] \ell^{*}, \ell_{0}[$ for all $t \in \mathbb{R}$. Moreover, if $j=0, \hat{x}(t)>\xi_{1}$ for all $t \in\left[t_{-}, t_{+}\right]$, while $\hat{x}(t)$ has precisely $2 j$ zeros in ]$t_{-}, t_{+}[$if $j \geq 1$.

Using the fact that the energy level lines of (2.7) in the region $\mathcal{W} \backslash\{O\}=\left\{(x, y):-\xi_{0} \leq\right.$ $\left.x \leq \xi_{0}, 0<E(x, y) \leq \ell_{0}\right\}$ are strictly star-shaped with respect to the origin and symmetric with respect to the $x$-axis, we find that (2.12) holds (for some nonnegative integer $j$ ) if and only if

$$
\theta(T, \hat{s})=-\theta(\hat{s})-2 j \pi
$$

is satisfied (for the same $j$ ).

By virtue of (2.13), we can refer only to the angular coordinates, hence we will obtain solutions as follows. Since the point $\Phi_{0}^{T}(\gamma(1))$ lies on $\Gamma^{\ell_{0}}$ (which is the trajectory of a heteroclinic solution of (2.7)), it can never reach the point $Q_{+}$. Accordingly, the angle $\theta(T, 1)$ satisfies

$$
\theta(T, 1)>0, \quad \forall T \geq 0 .
$$


On the other hand, as $\gamma(0)=P_{+}$, we have that $\theta(0,0)=\theta(0)=0$, therefore

$$
\theta(T, 0)<0, \quad \forall T>0 \text {. }
$$

As a consequence, $\theta(T, 0)+\theta(0)<0<\theta(T, 1)+\theta(1)$ and the intermediate value theorem ensures the existence of an $\hat{s}$ such that $\theta(T, \hat{s})=-\theta(\hat{s})$. In this way we have found a positive homoclinic solution to $P_{+}$, independently of the length $T$ of the time interval $\left[t_{-}, t_{+}\right]$.

Suppose now that $T>n \tau_{\ell^{*}}$ for some positive integer $n$. In this case,

$$
\theta(T, 0)<-2 n \pi, \quad \forall T>0 .
$$

As a consequence, for every integer $j$ with $1 \leq j \leq n$, we have $\theta(T, 0)+\theta(0)<-2 j \pi<0<$ $\theta(T, 1)+\theta(1)$, and again the intermediate value theorem ensures the existence of an $\hat{s}$ such that $\theta(T, \hat{s})=-\theta(\hat{s})-2 j \pi$. This ends the proof for homoclinic solutions.

For the search of heteroclinic solutions from $P_{+}$to $P_{-}$, we follow a similar procedure, choosing as a target the set $y^{\prime \prime}$, which is the symmetric of $\mathcal{y}$ with respect to the $y$-axis (here we exploit the oddness of the nonlinear term $x^{3}$ ). In terms of polar coordinates, the desired solutions will be obtained if and only if there exists $\hat{s} \in] 0,1[$ such that

$$
\theta(T, \hat{s})=-\theta(\hat{s})-(2 j-1) \pi
$$

for some integer $j \geq 1$. Then the proof can be concluded as above, via the intermediate value theorem. We observe that the case of heteroclinic solutions from $P_{-}$to $P_{+}$is analogous, thus it will be omitted.

The next theorem deals with the internal connections. For this result, it is useful to recall the rotational gap defined in (2.11).

Theorem 2.2 (Internal connections) Under the above assumptions, the following results hold for solutions which satisfy the energy condition

$$
x^{\prime}(t)^{2}+2 k x(t)^{2}-\mu_{0} x(t)^{4} \in\left[\ell_{1}, \ell^{*}\right], \quad \forall t \in \mathbb{R}:
$$

- there always exist a solution homoclinic to $P_{+}$and a solution homoclinic to $P_{-}$;

- there always exist a heteroclinic solution from $P_{+}$to $P_{-}$and a heteroclinic solution from $P_{-}$to $P_{+}$;

- if $t_{+}-t_{-}>\frac{\tau_{\ell_{1}} \tau_{\ell^{*}}}{\tau_{\ell^{*}-\tau_{\ell_{1}}}}$, there exist at least $2 w\left(t_{+}-t_{-}\right)+1$ solutions homoclinic to $P_{+}$and $2 w\left(t_{+}-t_{-}\right)+1$ solutions homoclinic to $P_{-}$;

- if $t_{+}-t_{-}>\frac{\tau_{\ell_{1}} \tau_{\ell^{*}}}{\tau_{\ell^{*}-\tau_{\ell_{1}}}}$, there exist at least $2 w\left(t_{+}-t_{-}\right)+1$ heteroclinic solutions from $P_{+}$to $P_{-}$ and $2 w\left(t_{+}-t_{-}\right)+1$ from $P_{-}$to $P_{+}$.

Proof As the first step, we focus our attention on the search of solutions homoclinic to the point $P_{+}$. The case of homoclinics to $P_{-}$is analogous, thus it will be omitted.

Our goal is to find points on $\mathcal{L}=\overline{\mathcal{L}\left(P_{+}, P_{-}\right)}$which are moved by the Poincaré map $\Phi_{0}^{T}$ on $\mathcal{L}^{\prime}=\overline{\mathcal{L}\left(P_{-}, P_{+}\right)}$.

As a preliminary remark, we note that $\Phi_{0}^{T}(\mathcal{L})$ is a simple arc contained in $\mathscr{E}$, connecting its end points $\Phi_{0}^{T}\left(P_{+}\right)$and $\Phi_{0}^{T}\left(P_{-}\right)$on $\Gamma^{\ell^{*}}$ through the 'intermediate' point $\Phi_{0}^{T}(R)$ on $\Gamma^{\ell_{1}}$. 
Observe also that $\Phi_{0}^{T}\left(P_{+}\right)$and $\Phi_{0}^{T}\left(P_{-}\right)$are antipodal. In fact, $P_{-}$and $P_{+}$are antipodal and $\Phi_{0}^{T}$ is an odd map (indeed, $(x(t), y(t))$ is a solution of (2.7) if and only if $(-x(t),-y(t))$ is a solution of the same equation). Now, if the trivial situation $\left\{\Phi_{0}^{T}\left(P_{-}\right), \Phi_{0}^{T}\left(P_{+}\right)\right\}=\left\{P_{-}, P_{+}\right\}$ occurs, we have $\Phi_{0}^{T}(\mathcal{L}) \cap \mathcal{L}^{\prime}=\left\{P_{-}, P_{+}\right\} \neq \emptyset$. Otherwise, we find that $\Phi_{0}^{T}\left(P_{-}\right)$and $\Phi_{0}^{T}\left(P_{+}\right)$ belong to the two different components of $\mathcal{E} \backslash \mathcal{L}^{\prime}$. Since $\Phi_{0}^{T}\left(P_{-}\right)$and $\Phi_{0}^{T}\left(P_{+}\right)$are the end points of the $\operatorname{arc} \Phi_{0}^{T}(\mathscr{L}) \subseteq \mathcal{E}$, by an elementary connectivity argument, we conclude that $\Phi_{0}^{T}(\mathcal{L}) \cap \mathcal{L}^{\prime} \neq \emptyset$. This proves the first assertion of the theorem.

For the search of heteroclinic solutions from $P_{+}$to $P_{-}$, we adopt a similar procedure, choosing as a target the set $\mathscr{L}$ itself. The fact that, for every $T>0$, there is always at least one intersection point between $\Phi_{0}^{T}(\mathscr{L})$ and $\mathscr{L}$ follows by the same argument developed for homoclinic solutions. Namely, if the trivial situation $\left\{\Phi_{0}^{T}\left(P_{-}\right), \Phi_{0}^{T}\left(P_{+}\right)\right\}=\left\{P_{-}, P_{+}\right\}$occurs, we have $\Phi_{0}^{T}(\mathcal{L}) \cap \mathcal{L}=\left\{P_{-}, P_{+}\right\} \neq \emptyset$. Otherwise, we find that $\Phi_{0}^{T}\left(P_{-}\right)$and $\Phi_{0}^{T}\left(P_{+}\right)$belong to the two different components of $\mathscr{E} \backslash \mathscr{L}$. Therefore, it follows that $\Phi_{0}^{T}(\mathcal{L}) \cap \mathscr{L} \neq \emptyset$.

We study now the problem of multiplicity of solutions. As before, we consider at first the case of solutions homoclinic to $P_{+}$.

We introduce in the inner region $\mathcal{E}$ of a phase-plane for (2.7) a system of modified polar coordinates $(\theta, E)$ with center in the origin. In this system, every point is determined by its angular coordinate $\theta$ and its energy $E$ defined in (2.8). Since the energy level lines in $\mathscr{E}$ are strictly star-shaped with respect to the origin, we obtain a coordinate system equivalent to the polar one.

Observe that the set $\mathcal{L} \cup \mathcal{L}^{\prime}$ intersects every half-line from the origin exactly in one point. Accordingly, we can parameterize those points using the angular coordinate. With this convention, the initial points in the arc $\mathcal{L}$ are parameterized as $\gamma(s)$ for $s \in[\pi, 2 \pi]$ ( $s$ is the angle) with $\gamma(\pi)=P_{-}, \gamma(2 \pi)=P_{+}$and $\gamma(3 \pi / 2)=R$. As a consequence, $E(\gamma(s))$ is strictly decreasing for $s \in[\pi, 3 \pi / 2]$ and strictly increasing in $[3 \pi / 2,2 \pi]$. The target set $\mathcal{L}^{\prime}$ (the symmetric of $\mathcal{L}$ with respect to the $x$-axis) is parameterized with the angle $s \in[0, \pi]$.

For the specific case of system (2.6), an analytic expression for $\mathcal{L}$ as a graph in the $(\theta, E)$ plane, is given as follows:

$$
\begin{aligned}
E(\theta) & :=E(\rho(\theta) \cos \theta, \rho(\theta) \sin \theta) \\
& =\rho^{2}(\theta) \sin ^{2} \theta+2 k \rho^{2}(\theta) \cos ^{2} \theta-\mu_{0} \rho^{4}(\theta) \cos ^{4} \theta,
\end{aligned}
$$

with

$$
\rho(\theta)=\frac{\left(2 k / \sqrt{\mu_{1}}\right)}{\left(\sin ^{2} \theta+4 k \cos ^{2} \theta\right)^{1 / 2}-\sin \theta}, \quad \theta \in[\pi, 2 \pi] .
$$

Hence, the natural parametrization of $\mathcal{L}$ in the $(\theta, E)$-plane is given by $\gamma(s)=(s, E(s))$ for $s=\theta \in[\pi, 2 \pi]$.

Using the same modified polar coordinates to represent the solutions of (2.7), we can express the final points $\Phi_{0}^{T}(\mathscr{L})$ in the $(\theta, E)$-plane by means of their angular coordinate $\theta(t, s)$ and energy $E(t, s)$. As in the previous proof, we observe that, for every $s \in[\pi, 2 \pi]$, the map $t \mapsto \theta(t, s)$ is strictly decreasing on $[0,+\infty)$, while the energy is constant with respect to $t$. Observe also that in the new coordinates $(\theta, E)$, system $(2.7)$ becomes

$$
\left\{\begin{array}{l}
\theta^{\prime}=-\sin ^{2} \theta-2 k \cos ^{2} \theta+2 \mu_{0} \Lambda(\theta, E) \cos ^{4} \theta \\
E^{\prime}=0
\end{array}\right.
$$


with

$$
\Lambda(\theta, E):=\frac{2 E}{\sin ^{2} \theta+2 k \cos ^{2} \theta+\left(\left(\sin ^{2} \theta+2 k \cos ^{2} \theta\right)^{2}-4 \mu_{0} E \cos ^{4} \theta\right)^{1 / 2}} .
$$

In the $(\theta, E)$-plane, the points of $\mathscr{L}$ move with a negative angular speed along the lines of constant energy. Thus, under the action of the flow associated to (2.16), the points of $\mathcal{L}$ shift from the right to the left in the strip

$$
\mathcal{E}(-\infty, 2 \pi]:=\left\{(\theta, E): \theta \leq 2 \pi, \ell_{1} \leq E \leq \ell^{*}\right\} .
$$

As we have explained before, we obtain an internal solution homoclinic to $P_{+}$whenever there is a point $z \in \mathscr{L}$ such that $\Phi_{0}^{T}(z) \in \mathscr{L}^{\prime}$, with $T=t_{+}-t_{-}$. In the $(\theta, E)$-plane, this target set is expressed as the union of the graphs

$$
\Xi:=\bigcup_{j=0}^{\infty}\{(\theta, \Upsilon(\theta)):-2 j \pi \leq \theta \leq \pi-2 j \pi\}
$$

where

$$
\Upsilon(\theta):=E(\varrho(\theta) \cos \theta, \varrho(\theta) \sin \theta)=\varrho^{2}(\theta) \sin ^{2} \theta+2 k \varrho^{2}(\theta) \cos ^{2} \theta-\mu_{0} \varrho^{4}(\theta) \cos ^{4} \theta,
$$

with

$$
\varrho(\theta)=\frac{\left(2 k / \sqrt{\mu_{1}}\right)}{\left(\sin ^{2} \theta+4 k \cos ^{2} \theta\right)^{1 / 2}+\sin \theta}
$$

(see Figures 10, 11 for a graphical description).

In this setting, we obtain an internal solution homoclinic to $P_{+}$if and only if there exists $\hat{s} \in[\pi, 2 \pi]$ such that $\Phi(\gamma(\hat{s})) \in \Xi$, where we have denoted by $\Phi$ the Poincaré map for (2.16) in the time interval $[0, T]$. Of course $\Phi$ is exactly $\Phi_{0}^{T}$ (which was the Poincaré map associated to $(2.7))$ in the new $(\theta, E)$-coordinates. Using the parameterized curve $\gamma(s)$ for the initial points, we can express $\Phi$ as

$$
\Phi(\gamma(s))=(\theta(T, s), E(s)) \quad \text { for } s \in[\pi, 2 \pi]
$$

(recall that $\gamma(s)=(s, E(s))$ for $s=\theta \in[\pi, 2 \pi])$.

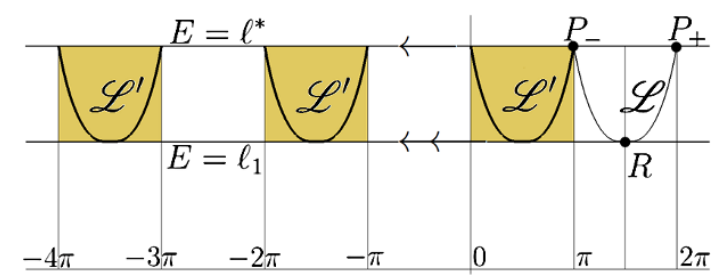

Figure 10 The initial set $\mathcal{L}$ and the target set $\mathcal{L}^{\prime}$ represented in the $(\theta, E)$-plane. The present figure is drawn for the parameters $k=1, \mu_{1}=2, \mu_{0}=1 / 2$. The internal region $\boldsymbol{E}$ corresponds to the strip $\mathbb{R} \times\left[\ell_{1}, \ell^{*}\right]$. Since we are interested in the evolution of the set $\mathscr{L}$ through the Poincare map, we consider only the half-strip $\boldsymbol{E}(-\infty, 2 \pi]=(-\infty, 2 \pi] \times\left[\ell_{1}, \ell^{*}\right]$. For the flow associated to (2.16), all the points of $\boldsymbol{\mathcal { L }}$ move from the right to the left on lines parallel to the $\theta$-axis. The point $R$ (as well as the points on the line $E=\ell_{1}$ ) moves faster than the points $P_{ \pm}$which are on the line $E=\ell^{*}$. During all the evolution, the distance between the images of $P_{-}$and $P_{+}$remains constantly equal to $\pi$. 


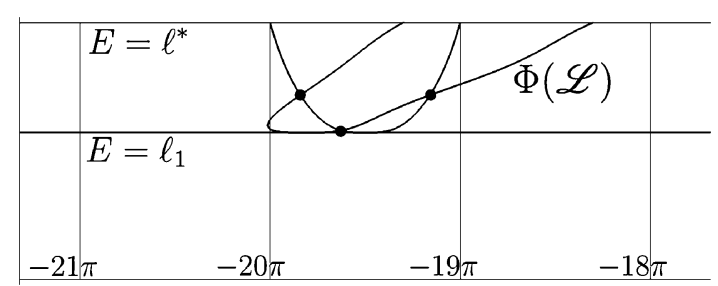

Figure 11 The present figure describes the same situation of Figure 7 in the angle-energy coordinates (with the same choice of the coefficients $k, \mu_{1}, \mu_{0}$ and time gap $T=t_{+}-t_{-}=50$ ).

The points $P_{-}$and $P_{+}$are antipodal, lie on the same energy line $\Gamma^{\ell^{*}}$ and move with the same angular speed. In the $(\theta, E)$-plane, $P_{-}$and $P_{+}$are expressed by $P_{-}=\left(\pi, \ell^{*}\right), P_{+}=$ $\left(2 \pi, \ell^{*}\right)$. Therefore,

$$
\theta(t, \pi)-\theta(t, 2 \pi)=-\pi, \quad \forall t \geq 0 .
$$

Moreover, for every integer $j \geq 0$, we have that

$$
\theta(t, \pi) \lesseqgtr \pi-j \pi \quad \text { if and only if } \quad t \gtreqless j \frac{\tau_{\ell^{*}}}{2}
$$

(this follows from the fact that the orbit $\Gamma^{\ell^{*}}$ has a period $\tau_{\ell^{*}}$ and is symmetric with respect to the $x$-axis).

In the same plane, the point $R$ is indicated by $R=\left(3 \pi / 2, \ell_{1}\right)$. Using the fact that the orbit $\Gamma^{\ell_{1}}$ has a period $\tau_{\ell_{1}}$ and is symmetric with respect to the $y$-axis, we find that, for every integer $i \geq 0$,

$$
\theta(t, 3 \pi / 2) \lesseqgtr \frac{\pi}{2}-2 i \pi \quad \text { if and only if } \quad t \gtreqless \frac{\tau_{\ell_{1}}}{2}+i \tau_{\ell_{1}} \text {. }
$$

Suppose now that

$$
\frac{2 T}{\tau_{\ell_{1}}}-\frac{2 T}{\tau_{\ell^{*}}}>2 .
$$

Then, the open interval $] 2 T / \tau_{\ell^{*}}, 2 T / \tau_{\ell_{1}}$ [ contains at least one odd integer.

Let $\mathbf{m}_{o}(T)$ be the largest odd integer contained in $] 2 T / \tau_{\ell^{*}}, 2 T / \tau_{\ell_{1}}$. From $T>\mathbf{m}_{o}(T) \tau_{\ell_{1}} / 2$, we have that

$$
\theta(T, 3 \pi / 2)<\frac{\pi}{2}-\left(\mathbf{m}_{o}(T)-1\right) \pi=\frac{3 \pi}{2}-\mathbf{m}_{o}(T) \pi .
$$

Suppose also that $j$ is a positive integer with $2 T / \tau_{\ell^{*}}<j \leq \mathbf{m}_{o}(T)$. In this case, from $T<$ $j \tau_{\ell^{*}} / 2$, we have that

$$
\theta(T, \pi)>\pi-j \pi \quad \text { and } \quad \theta(T, 2 \pi)>2 \pi-j \pi .
$$

Let

$$
\mathcal{L}_{\text {left }}:=\{(\theta, E(\theta)): \pi \leq \theta \leq 3 \pi / 2\}
$$


and

$$
\mathcal{L}_{\text {right }}:=\{(\theta, E(\theta)): 3 \pi / 2 \leq \theta \leq 2 \pi\}
$$

with $\mathcal{L}=\mathscr{L}_{\text {left }} \cup \mathcal{L}_{\text {right }}$ be the left and the right parts of $\mathcal{L}$ in the $(\theta, E)$-plane. Similarly, we define

$$
\mathcal{L}_{\text {left }}^{\prime}:=\{(\theta, \Upsilon(\theta)): 0 \leq \theta \leq \pi / 2\}
$$

and

$$
\mathcal{L}_{\text {right }}^{\prime}:=\{(\theta, \Upsilon(\theta)): \pi / 2 \leq \theta \leq \pi\}
$$

By definition, for any nonnegative integer $i$, the set $\mathcal{L}_{\text {left }}^{\prime}-(2 i \pi, 0)$ is a simple arc connecting the points $\left(-2 i \pi, \ell^{*}\right)$ and $\left(-2 i \pi+(\pi / 2), \ell_{1}\right)$ in the rectangle $[-2 i \pi,-2 i \pi+(\pi / 2)] \times\left[\ell_{1}, \ell^{*}\right]$ and, similarly, $\mathcal{L}_{\text {right }}^{\prime}-(2 i \pi, 0)$ is a simple arc connecting the points $\left(-2 i \pi+(\pi / 2), \ell_{1}\right)$ and $\left(-2 i \pi+\pi, \ell^{*}\right)$ in the rectangle $[-2 i \pi+(\pi / 2),-2 i \pi+\pi] \times\left[\ell_{1}, \ell^{*}\right]$. On the other hand, the set $\Phi\left(\mathcal{L}_{\text {left }}\right)$ is a simple arc connecting the points $\left(\theta(T, \pi), \ell^{*}\right)$ and $\left(\theta(T, 3 \pi / 2), \ell_{1}\right)$ in $\mathcal{E}(-\infty, 2 \pi]$ and, similarly, $\Phi\left(\mathcal{L}_{\text {right }}\right)$ is a simple arc connecting the points $\left(\theta(T, 3 \pi / 2), \ell_{1}\right)$ and $\left(\theta(T, 2 \pi), \ell^{*}\right)=\left(\pi+\theta(T, \pi), \ell^{*}\right)$ in the same strip.

Suppose now that $j=\mathbf{m}_{o}(T)=2 k+1$ (in this case, $j$ is odd). In such a situation, from (2.19) and (2.20) we have that

$$
\theta(T, 3 \pi / 2)<\frac{\pi}{2}-2 k \pi, \quad \theta(T, \pi)>-2 k \pi, \quad \theta(T, \pi)>\pi-2 k \pi,
$$

for $2 k=j-1$ an even integer. Hence the points $\left(\theta(T, 3 \pi / 2), \ell_{1}\right)$ and $\left(\theta(T, \pi), \ell^{*}\right)$ are separated by the arc $\mathcal{L}_{\text {left }}^{\prime}-(2 k \pi, 0)$, while the points $\left(\theta(T, 3 \pi / 2), \ell_{1}\right)$ and $\left(\theta(T, 2 \pi), \ell^{*}\right)$ are separated by $\mathscr{L}_{\text {left }}^{\prime}-(2 k \pi, 0)$ and $\mathcal{L}_{\text {right }}^{\prime}-(2 k \pi, 0)$. As a consequence, $\Phi(\mathscr{L}) \cap \Xi$ contains at least three points, precisely the nonempty intersections of $\Phi\left(\mathcal{L}_{\text {left }}\right) \cap\left(\mathcal{L}_{\text {left }}^{\prime}-(2 k \pi, 0)\right)$, $\Phi\left(\mathcal{L}_{\text {right }}\right) \cap\left(\mathcal{L}_{\text {left }}^{\prime}-(2 k \pi, 0)\right)$ and $\Phi\left(\mathcal{L}_{\text {right }}\right) \cap\left(\mathcal{L}_{\text {right }}^{\prime}-(2 k \pi, 0)\right)$.

Suppose that $j=\mathbf{m}_{o}(T)-1$ (in this case, $j$ is even). In such a situation, from (2.19) and (2.20) we have that

$$
\theta(T, 3 \pi / 2)<\frac{\pi}{2}-2 k \pi, \quad \theta(T, \pi)>\pi-2 k \pi, \quad \theta(T, \pi)>2 \pi-2 k \pi
$$

for $2 k=\mathbf{m}_{o}(T)-1$ an even integer. Hence the points $\left(\theta(T, 3 \pi / 2), \ell_{1}\right)$ and $\left(\theta(T, \pi), \ell^{*}\right)$ are separated by the arcs $\mathcal{L}_{\text {left }}^{\prime}-(2 k \pi, 0)$ and $\mathcal{L}_{\text {right }}^{\prime}-(2 k \pi, 0)$. The points $\left(\theta(T, 3 \pi / 2), \ell_{1}\right)$ and $\left(\theta(T, 2 \pi), \ell^{*}\right)$ are separated by $\mathcal{L}_{\text {left }}^{\prime}-(2 k \pi, 0), \mathcal{L}_{\text {right }}^{\prime}-(2 k \pi, 0)$ and $\mathcal{L}_{\text {left }}^{\prime}-((2 k-1) \pi, 0)$. As a consequence, $\Phi(\mathscr{L}) \cap \Xi$ contains at least five points: two points coming from the intersections of $\Phi\left(\mathcal{L}_{\text {left }}\right)$ with the target set $\Xi$ and three from the intersections of $\Phi\left(\mathcal{L}_{\text {right }}\right)$.

Proceeding by induction, with the same argument, the following result is obtained.

Claim $1 \Phi(\mathcal{L}) \cap \Xi$ has at least $2 n^{\#}+1$ solutions, where $n^{\#}$ is the number of integers less than or equal to $\mathbf{m}_{o}(T)$ which are contained in the open interval $] 2 T / \tau_{\ell^{*}}, 2 T / \tau_{\ell_{1}}[$.

A completely symmetric argument leads to the same multiplicity result for solutions which are homoclinic to $P_{-}$. 
At last, we look for a multiplicity result for heteroclinic solutions from $P_{+}$to $P_{-}$. We follow step by step the argument described in the part of the proof devoted to the search of multiple internal homoclinic solutions and, therefore, we transform our equation in the $(\theta, E)$-coordinates. As we have explained before, we obtain an internal heteroclinic (from $P_{+}$to $\left.P_{-}\right)$whenever there is a point $z \in \mathcal{L}$ such that $\Phi_{0}^{T}(z) \in \mathcal{L}$, with $T=t_{+}-t_{-}$. In the $(\theta, E)$-plane, this target set is expressed as the union of the graphs

$$
\Xi^{\prime}:=\bigcup_{j=0}^{\infty}\{(\theta, E(\theta)): \pi-2 j \pi \leq \theta \leq 2 \pi-2 j \pi\},
$$

where $E(\theta)$ is the function defined in (2.15).

As before, we assume the validity of condition (2.18). Then the open interval $] 2 T / \tau_{\ell^{*}}$, $2 T / \tau_{\ell_{1}}$ [ contains at least one even integer.

Let $\mathbf{m}_{e}(T)$ be the largest even integer contained in $] 2 T / \tau_{\ell^{*}}, 2 T / \tau_{\ell_{1}}\left[\right.$. From $T>\mathbf{m}_{e}(T) \tau_{\ell_{1}} /$ 2 , we have that

$$
\theta(T, 3 \pi / 2)<\frac{3 \pi}{2}-\mathbf{m}_{e}(T) \pi
$$

For any positive integer $j$ with $2 T / \tau_{\ell^{*}}<j \leq \mathbf{m}_{e}(T)$, we have that (2.20) holds too. At this point, we have simply to repeat (with obvious changes due to the fact that the target set is $\Xi^{\prime}$ which is a shift of $\Xi$ by $\left.(2 p i, 0)\right)$ the argument presented above for the case of homoclinic connections and obtain the following conclusion.

Claim $2 \Phi(\mathcal{L}) \cap \Xi^{\prime}$ has at least $2 n_{\#}+1$ solutions, where $n_{\#}$ is the number of integers less than or equal to $\mathbf{m}_{e}(T)$ which are contained in the open interval $] 2 T / \tau_{\ell^{*}}, 2 T / \tau_{\ell_{1}}[$.

A completely symmetric argument leads to a multiplicity result for heteroclinic solutions from $P_{-}$to $P_{+}$.

As final step, we have just to show how the condition on the rotational gap allows us to achieve the conclusion from Claim 1 and Claim 2 that we have obtained along the proof. Now, it is sufficient to observe that $t_{+}-t_{-}=T>\frac{\tau_{\ell_{1}} \tau_{\ell^{*}}}{\tau_{\ell^{*}-\tau_{\ell_{1}}}}$ implies (2.18) and, therefore, the open interval ] $2 T / \tau_{\ell^{*}}, 2 T / \tau_{\ell_{1}}$ [ contains at least two integers. More precisely, by the definition of $w(T)$, the interval $] 2 T / \tau_{\ell^{*}}, 2 T / \tau_{\ell_{1}}$ [ contains at least $w(T)+1$ integers and, therefore,

$$
n^{\#}, n_{\#} \geq w(T)
$$

This concludes the proof of the theorem.

\section{Remarks and related results}

We end the paper with a list of remarks about possible variants and extensions of the main results obtained for equation

$$
x^{\prime \prime}+2 k x-2 g(t) x^{3}=0 .
$$

1. A problem which naturally arises from the analysis that we have performed concerns what happens if, for a stepwise weight function satisfying (2.5), we suppose that 
$0<\mu_{1}<\mu_{0}$. Repeating the preliminary phase-plane analysis of Section 2.2, one can easily check that for any gap $T=t_{+}-t_{-}>0$, there always exist a solution homoclinic to $P_{+}$and another homoclinic to $P_{-}$, as well as a heteroclinic from $P_{+}$to $P_{-}$and another one from $P_{-}$to $P_{+}$. However, in general, if $0<\mu_{1}<\mu_{0}$, one cannot obtain multiplicity results like those achieved in Section 2 without some extra assumptions on $h(x)$. This is the reason for which, in the study of equation (2.1), we have considered only the case $0<\mu_{0}<\mu_{1}$. For different examples on related equations in which a weight coefficient can be above or below its limits at infinity, see, for instance, [4-6].

On the other hand, if we assume that the weight function changes its sign, with $\mu_{0}<0<\mu_{1}$, some interesting multiplicity results could be produced. In fact the phase portrait of (1.1) in the time interval $\left[t_{-}, t_{+}\right]$shows a global center, hence if $t_{+}-t_{-}$is large, a lot of connections between the unstable and stable manifolds of the points $P_{ \pm}$ can be obtained. For the sake of conciseness, we omit the study of this latter situation, which is beyond the goal of the present paper.

2. We notice that the same argument of the proofs applies to an equation of the form

$$
x^{\prime \prime}+\sigma x-a(t) h(x)=0
$$

for $\sigma>0, h(x)=x f(x)$, and with $f(x)$ satisfying condition $(*)$, if we assume that $a(t)$ is a stepwise weight function, playing the same role of $g(t)$ in (2.1). In such a case, the saddle points become $P_{ \pm}=\left( \pm \xi_{1}, 0\right)$ with $\xi_{1}:=f^{-1}\left(\sigma / \mu_{1}\right)$, where $f^{-1}$ is the inverse of $f$ restricted to $[0,+\infty)$. In this way, we can apply our results to nonlinearities like those considered in [2, 27]. More precisely, Theorem 2.1 holds without any further assumption, while for Theorem 2.2 we need to require a gap between the periods of the orbits $\Gamma^{\ell_{1}}$ and $\Gamma^{\ell^{*}}$. In order to obtain this gap, we can apply (for instance) some results ensuring the monotonicity of the time-map (like [28, Theorem A]).

3. Due to the special form of the weight coefficient, it is standard to verify (via a simple rescaling procedure) that (1.1) is equivalent to an equation of the form

$$
x^{\prime \prime}+b(t) x-a h(x)=0
$$

where $a>0$ and $b(t)$ is a stepwise coefficient. In this manner, one can deal with some nonlinear Schrödinger-type equations related to the case of potential wells or potential walls $[29,30]$.

4. The approach used in the proofs, based on the properties of the Poincare map, guarantees that our results are stable with respect to small perturbations. More precisely, fixed a suitable length $T=t_{+}-t_{-}$for the time interval, Theorems 2.1 and 2.2 provide a lower bound for the number of solutions. We can state that the same lower bound persists for a small perturbation of the coefficient in the $L^{1}$-norm on $\left[t_{-}, t_{+}\right]$. Therefore, the assumption that the weight $a(t)$ in (1.1) or $b(t)$ in (3.1) are stepwise functions can be slightly relaxed, so we can 'smooth' them.

5. With reference to equations (1.1) or (3.1) with stepwise coefficients, we observe that our approach can be adapted to boundary value problems on a compact interval $[0, T]$ like, e.g., the Dirichlet (two-point) or the Neumann problem. In these cases, we have to find solutions connecting given lines which depart from the origin. For the 
Figure 12 In the present figure the line $\Upsilon^{*}$ is the transformation of the segment $\left[-\xi_{1}^{*}, \xi_{1}^{*}\right]$ $\times\{0\}$ under the Poincaré map $\Phi_{1}$ for $\tau=2.2$. The parameters used are $k=1, \mu_{1}=2, \mu_{0}=1 / 2$. For graphical reasons, a slightly different $x$ - and $y$-scaling has been used.

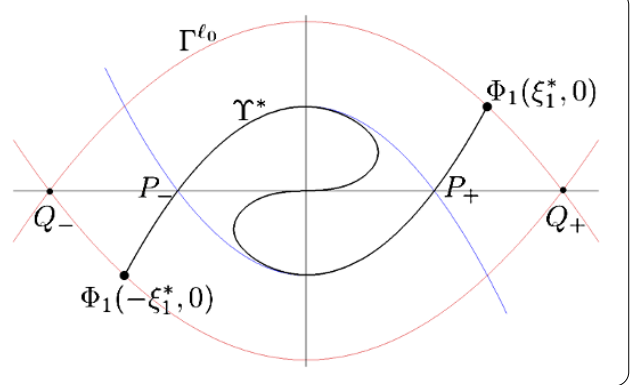

sake of conciseness, we cannot describe the most general situation, but we just outline a possible application for the Neumann problem

$$
\left\{\begin{array}{l}
x^{\prime \prime}+2 k x-2 g(t) x^{3}=0, \\
x^{\prime}(0)=0, \quad x^{\prime}(T)=0,
\end{array}\right.
$$

with $g(t)$ a stepwise function such that $g(t)=\mu_{1}$ for $t \in\left[0, \tau\left[\right.\right.$ and $g(t)=\mu_{0}$ for $t \in[\tau, T]$, for $0<\mu_{0}<\mu_{1}$. Let us denote by $\Phi_{1}$ the Poincaré map associated to system (2.6) on the time interval $[0, \tau]$, and by $\Phi_{2}$ the Poincaré map associated to system (2.7) on the time interval $[0, T-\tau]$. We observe that there exists a maximal compact interval $\left[-\xi_{1}^{*}, \xi_{1}^{*}\right]$ with $\xi_{1}<\xi_{1}^{*}<\xi_{0}$ such that $E\left(\Phi_{1}(z)\right) \leq \ell_{0}$ for all the points $z=(x, 0)$ with $x \in\left[-\xi_{1}^{*}, \xi_{1}^{*}\right]$. In this manner $\Phi_{1}\left( \pm \xi_{1}^{*}, 0\right) \in \Gamma^{\ell_{0}}$. The curve

$$
\Upsilon^{*}:=\left\{\Phi_{1}(x, 0):-\xi_{1}^{*} \leq x \leq \xi_{1}^{*}\right\}
$$

represents the set of all the points in the region $\mathcal{W}=\left\{(x, y):-\xi_{0} \leq x \leq \xi_{0}\right.$, $\left.E(x, y) \leq \ell_{0}\right\}$, which are images (by $\Phi_{1}$ ) of initial points of the $x$-axis (therefore $\left.x^{\prime}(0)=0\right)$. Figure 12 illustrates the curve $\Upsilon^{*}$ for a short time interval $(\tau=2.2)$. For a larger $\tau$, the line $\Upsilon^{*}$ becomes a double spiral with a certain number of turns around the origin, while the part of $\Upsilon^{*}$ contained in the region $\mathcal{F}$ gets very close to $\mathcal{O}\left(P_{+}\right)$ and $\mathcal{O}\left(P_{-}\right)$. Then we can repeat the same argument developed in the previous sections by looking for the intersections of $\Phi_{2}\left(\Upsilon^{*}\right)$ with the $x$-axis.

6. As a final remark, we mention the fact that combining our technique with Ważewski's method [31], following the approach developed by Conley in [18], one can deal with some more general classes of weight functions. For example, one could tackle with these techniques the cases of asymptotically constant or asymptotically periodic coefficients. These extensions, however, need a more delicate analysis and they are beyond the goals of the present paper.

We hope that the abundance of multiplicity results found in the present work (in the special case of stepwise coefficients) may suggest possible directions for extending Theorem 2.1 and Theorem 2.2 to more general weight functions. This will be our goal for a future investigation of the problem.

Competing interests

The authors declare that they have no competing interests. 


\section{Acknowledgements}

The authors are deeply indebted with the referee for the careful checking of the manuscript and for his/her remarks, including a correction to an erroneous argument in the previous version of the proof. This research was partially supported by the project PRIN-2009 Equazioni Differenziali Ordinarie e Applicazioni.

Received: 30 November 2012 Accepted: 11 June 2013 Published: 15 July 2013

\section{References}

1. Belmonte-Beitia, J, Torres, PJ: Existence of dark soliton solutions of the cubic nonlinear Schrödinger equation with periodic inhomogeneous nonlinearity. J. Nonlinear Math. Phys. 15(3), 65-72 (2008)

2. Torres, PJ, Konotop, V: On the existence of dark solitons in a cubic-quintic nonlinear Schrödinger equation with a periodic potential. Commun. Math. Phys. 282, 1-9 (2008)

3. Ambrosetti, A, Badiale, M, Cingolani, S: Semiclassical states of nonlinear Schrödinger equations. Arch. Ration. Mech. Anal. 140, 285-300 (1997)

4. Gavioli, A: On the existence of heteroclinic trajectories for asymptotically autonomous equations. Topol. Methods Nonlinear Anal. 34, 251-266 (2009)

5. Gavioli, A: Monotone heteroclinic solutions to non-autonomous equations via phase plane analysis. Nonlinear Differ. Equ. Appl. 18, 79-100 (2011)

6. Gavioli, A, Sanchez, L: Heteroclinics for non-autonomous second-order differential equations. Differ. Integral Equ. 22 , 999-1018 (2009)

7. Holmes, PJ, Stuart, CA: Homoclinic orbits for eventually autonomous planar flows. Z. Angew. Math. Phys. 43, 598-625 (1992)

8. Jones, CKRT, Küpper, T, Schaffner, K: Bifurcation of asymmetric solutions in nonlinear optical media. Z. Angew. Math. Phys. 52, 859-880 (2001)

9. Aronson, DG, Mantzaris, NV, Othmer, HG: Wave propagation and blocking in inhomogeneous media. Discrete Contin. Dyn. Syst. 13, 843-876 (2005)

10. Lewis, TJ, Keener, JP: Wave-block in excitable media due to regions of depressed excitability. SIAM J. Appl. Math. 61, 293-316 (2000)

11. Yang, J, Kalliadasis, S, Merkin, JH, Scott, SK: Wave propagation in spatially distributed excitable media. SIAM J. Appl. Math. 63, 485-509 (2002)

12. Rodrigues, AS, Kevrekidis, PG, Porter, MA, Frantzeskakis, DJ, Schmelcher, P, Bishop, AR: Matter-wave solitons with a periodic, piecewise-constant scattering length. Phys. Rev. A 78, 013611 (2008)

13. Margheri, A, Rebelo, C, Zanolin, F: Connected branches of initial points for asymptotic BVPs, with application to heteroclinic and homoclinic solutions. Adv. Nonlinear Stud. 9, 95-135 (2009)

14. Boscaggin, A, Zanolin, F: Pairs of nodal solutions for a class of nonlinear problems with one-sided growth conditions. Adv. Nonlinear Stud. 13, 13-55 (2013)

15. Capietto, A, Henrard, M, Mawhin, M, Zanolin, F: A continuation approach to some forced superlinear Sturm-Liouville boundary value problems. Topol. Methods Nonlinear Anal. 3, 81-100 (1994)

16. Fonda, A, Garrione, M: Generalized Sturm-Liouville boundary conditions for first order differential systems in the plane. Topol. Methods Nonlinear Anal. (to appear)

17. Rebelo, C, Zanolin, F: On the existence and multiplicity of branches of nodal solutions for a class of parameter-dependent Sturm-Liouville problems via the shooting map. Differ. Integral Equ. 13, 1473-1502 (2000)

18. Conley, C: An application of Wazewski method to a non-linear boundary value problem which arises in population genetics. J. Math. Biol. 2, 241-249 (1975)

19. Rebelo, C, Sanchez, L: Existence and multiplicity for an O.D.E. with nonlinear conditions. Differ. Equ. Dyn. Syst. 3, 383-396 (1995)

20. Struwe, M: Multiple solutions of anticoercive boundary value problems for a class of ordinary differential equations of second order. J. Differ. Equ. 37, 285-295 (1980)

21. Gaines, RE, Mawhin, J: Ordinary differential equations with nonlinear boundary conditions. J. Differ. Equ. 26, 200-222 (1977)

22. Gaines, RE, Mawhin, J: Coincidence Degree, and Nonlinear Differential Equations. Lecture Notes in Mathematics, vol. 568. Springer, Berlin (1977)

23. Hale, JK: Ordinary Differential Equations, 2nd edn. Krieger, Huntington (1980)

24. Zanini, C, Zanolin, F: An example of chaos for a cubic nonlinear Schrödinger equation with periodic inhomogeneous nonlinearity. Adv. Nonlinear Stud. 12, 481-499 (2012)

25. Gradshteyn, IS, Ryzhik, IM: Table of Integrals, Series, and Products, 7th edn. Translation Edited and with a Preface by Alan Jeffrey. Academic Press, Boston (2007)

26. Meyer, KR: Jacobi elliptic functions from a dynamical systems point of view. Am. Math. Mon. 108, 729-737 (2001)

27. Belmonte-Beitia, J, Cuevas, J: Existence of dark solitons in a class of stationary nonlinear Schrödinger equations with periodically modulated nonlinearity and periodic asymptotics. J. Math. Phys. 52, 032702 (2011)

28. Chicone, C: The monotonicity of the period function for planar Hamiltonian vector fields. J. Differ. Equ. 69, $310-321$ (1987)

29. Frauenkron, H, Grassberger, P: Interaction of nonlinear Schrödinger solitons with an external potential. J. Phys. A 28, 4987-4996 (1995)

30. Sakaguchi, H, Tamura, M: Scattering and trapping of nonlinear Schrödinger solitons in external potentials. J. Phys. Soc. Jpn. 73, 503-506 (2004)

31. Ważewski, T: Sur un principe topologique de l'examen de l'allure asymptotique des intégrales des équations différentielles ordinaires. Ann. Soc. Polon. Math. 20, 279-313 (1947)

doi:10.1186/1687-2770-2013-167

Cite this article as: Ellero and Zanolin: Homoclinic and heteroclinic solutions for a class of second-order non-autonomous ordinary differential equations: multiplicity results for stepwise potentials. Boundary Value Problems 2013 2013:167. 